La

Révolution

française

\section{La Révolution française}

Cahiers de l'Institut d'histoire de la Révolution française

$3 \mid 2012$

Les comités des assemblées révolutionnaires : des laboratoires de la loi

\title{
Le Comité des colonies
}

Une institution au service de la « famille coloniale » ? (1789-1793)

The Colonial Committee: an institution in the service of the " colonial family "?

(1789-1793)

\section{Manuel Covo}

\section{(2) OpenEdition}

\section{Journals}

Édition électronique

URL : http://journals.openedition.org//rf/692

DOI : $10.4000 /$ Irf.692

ISSN : 2105-2557

Éditeur

IHMC - Institut d'histoire moderne et contemporaine (UMR 8066)

Référence électronique

Manuel Covo, "Le Comité des colonies », La Révolution française [En ligne], 3 | 2012, mis en ligne le 20 décembre 2012, consulté le 30 avril 2019. URL : http://journals.openedition.org//rf/692 ; DOI : 10.4000/Irf.692

Ce document a été généré automatiquement le 30 avril 2019.

(C) La Révolution française 


\title{
Le Comité des colonies
}

\author{
Une institution au service de la « famille coloniale »? (1789-1793) \\ The Colonial Committee: an institution in the service of the " colonial family "? \\ (1789-1793)
}

Manuel Covo

1 Le Comité des colonies fut sans doute le plus contesté de tous les comités sous l'Assemblée constituante. Aux yeux des Amis des Noirs, il passait pour le gardien du système colonial dans son ensemble et fut accusé d'avoir tâché de préserver durablement l'esclavage, la traite négrière et le préjugé de couleur. Mais si Brissot n'eut de cesse de protester contre « l'absurde système qui [avait] dirigé le comité colonial »" les critiques ne provinrent pas seulement de "philanthropes » réclamant la suppression du «commerce honteux » et l'abolition graduelle. L'avocat des colons, Linguet, considérait ainsi que de tous les comités «qui subjugu[aient] l'Assemblée nationale, il n'y en a[vait] point de plus dangereux, de plus coupable, de plus ignorant que celui des colonies $»^{2}$; le député de Nantes et négociant, Blin, dénonça la conversion du «comité colonial en ministre et ministère des colonies $\|^{3}$ tandis que Regnaud de Saint Jean d'Angély estimait que les colonies n'avaient «été troublées que parce que le Comité colonial de l'Assemblée nationale n'a[vait] pas été un comité constitutionnel qui se borne[rait] à préparer les travaux, mais un Comité administratif qui v[oulai]t régir $»^{4}$. D'un bout à l'autre du spectre politique, le Comité en vint à incarner la corruption de la Révolution en cours. La volonté générale s'était vue contrariée par la subordination systématique de l'Assemblée, l'accaparement du pouvoir législatif et la captation de la puissance exécutive.

2 A ce titre, c'est sur le rapporteur du Comité des colonies, Barnave, que se concentrèrent la plupart des attaques. Si l'aile gauche des "patriotes » pouvait s'attendre à ce que les « aristocrates » défendissent le système colonial, elle ne put tolérer la «trahison » d'un « révolutionnaire » qui, en 1789, voulait faire de la Déclaration des droits de l'homme et du citoyen le «catéchisme national ». Apparaissant progressivement comme le porteparole de l'esclavagisme et du préjugé de couleur, Barnave fut baptisé « Monsieur double visage », car, en «Janus », il imposa le principe du dualisme juridique contre la logique de l' « adunation $»^{5}$. A l'instar des contemporains, les historiens peinent toutefois à articuler 
l'influence réciproque de Barnave et du lobby colonial : le club Massiac ${ }^{6}$. Formé en août 1789 par une nébuleuse de planteurs et de négociants prêts à tout pour empêcher l'application de la Déclaration des droits de l'homme dans les colonies, ce groupe de pression aurait fait du Comité des colonies son cheval de Troie dans l'Assemblée ${ }^{7}$. Selon cette double historiographie, le comité pourrait apparaître comme un non-lieu dans la fabrique de la loi coloniale, d'autant que ses projets de décrets consistaient pour l'essentiel à limiter le pouvoir législatif de la représentation nationale au profit d'assemblées coloniales qui devaient disposer du monopole de l'initiative pour leur «régime intérieur ». Instrument d'un homme ou d'un groupe d'intérêt, le Comité ne serait rien d'autre qu'un paravent abritant des intrigues factieuses.

3 Le présent article, sans récuser ces interprétations, se propose de repenser le fonctionnement du Comité en reformulant le questionnement. En premier lieu, il s'agit de prendre l'existence du Comité au sérieux, de le constituer comme objet d'analyse pertinent, le seul travail d'envergure à son sujet ayant eu tendance à diluer le propos dans une réflexion générale sur la politique coloniale de l'Assemblée nationale ${ }^{8}$. Deuxièmement, nous ne limitons pas la réflexion au seul Comité de la Constituante, qui a complètement éclipsé les comités des assemblées suivantes dans l'historiographie' ${ }^{9}$ mais nous l'intégrons à un cadre chronologiquement plus large, en étudiant ses transformations sous la Législative et la Convention - jusqu'à sa fusion avec le Comité de marine en août 1793. De ce point de vue, cet objet offre un autre découpage temporel que celui qui domine l'historiographie, dont la problématique centrale interroge la « rupture républicaine » comme passage du dualisme juridique à la théorie de l'assimilation ${ }^{10}$. Enfin, c'est la spécificité des logiques coloniales qui est soumise à examen: la mise en quarantaine de la question coloniale, progressivement levée en France depuis les travaux d'Yves Bénot, a longtemps empêché de voir le rôle majeur joué par les colonies dans le processus révolutionnaire français ${ }^{11}$. Or, il est permis de s'interroger sur une possible «banalité » du Comité des colonies : bien avant la mise en place du Comité de salut public, ne serait-il que la caricature du comité révolutionnaire?

\section{La formation difficile du Comité des colonies}

4 La création d'un Comité des colonies n'allait pas de soi pour deux raisons : d'une part, elle induisait que les colonies devaient bénéficier d'un traitement législatif distinct de celui de la métropole et donc d'un système juridique dérogatoire; d'autre part, elle avalisait la compétence légiférante de l'Assemblée nationale sur les colonies. L'une et l'autre de ces propositions furent âprement discutées. Pour bien le comprendre, il faut se situer en amont et remettre la création du Comité dans le contexte spécifique de l'affrontement des groupes de pression coloniaux ${ }^{12}$. Le Comité fut en quelque sorte le fruit d'une tentative d'externalisation du domaine colonial hors du regard direct de l'Assemblée.

5 Son existence est d'abord liée à la question fondamentale de la représentation coloniale. Le roi avait refusé de convoquer des députés des colonies aux États généraux. Cependant, une majorité de grands planteurs blancs s'était néanmoins réunie en secret pour élire des représentants, chargés de s'introduire malgré tout à Versailles. A Paris, ils étaient appuyés par un premier groupe de pression, constitué essentiellement de propriétaires absents parisiens. Autour de son principal membre, Gouy d'Arsy, se forma une commission de Saint-Domingue chargée d'appuyer les demandes des colons. L'habile participation de ces planteurs au serment du Jeu de Paume permit de poser la question de 
leur admission à l'Assemblée nationale. Les députés voulaient y entrer afin de faire triompher leur projet autonomiste. Malgré l'opposition de Mirabeau, Ami des Noirs, et du député de Bordeaux Nairac, défendant les intérêts des négociants, la députation réussit à se faire admettre, le 3 juillet 1789. Cette représentation s'avéra en ce sens être un " piège » dans la mesure où elle soumettait les colonies au regard des constituants ${ }^{13}$.

En août 1789, la situation se compliqua pour les colons, alors que se profilait une Déclaration des droits de l'homme, en contradiction évidente avec le système colonial. En outre, la question avait suscité l'ébullition des lobbies : les Amis des Noirs de Brissot qui cherchaient à abolir la traite négrière, mais aussi les députés extraordinaires des manufactures et du commerce représentant les Chambres de commerce des ports, la société des libres de couleur autour de Julien Raymond, et enfin le club Massiac regroupant de riches colons et négociants ${ }^{14}$. Ce dernier club, fondé le 20 août 1789 , s'était donné pour objectif d'empêcher l'application des droits de l'homme dans les colonies, en tâchant de mettre en œuvre une stratégie commune aux intérêts des "coloniaux ». Il s'agissait donc d'organiser la résistance aux projets des députés-Amis des Noirs, tels que Grégoire, Pétion ou Mirabeau. La question de la formation d'un Comité des colonies se trouvait dès lors posée. Cependant le club Massiac ne parvint pas à coordonner complètement les interventions, alors que des rivalités personnelles avec les députés de Saint-Domingue mettaient ses stratégies à l'épreuve.

7 Dans un premier temps, le club Massiac était en effet hostile à la création d'un comité et espérait que l'Assemblée "oublierait» les colonies à la faveur de l'immensité de son travail. Cependant, les débats très virulents entre les députés des colons et les négociants sur la réforme de l'Exclusif colonial brisèrent le silence dès la fin août 1789. A cette occasion, il fut alors décrété que les colonies seraient « exceptées de la loi » générale ${ }^{15}$. Le 4 septembre, l'Assemblée ordonna la formation d'une commission chargée d'instruire la question. Les six membres de cette commission devaient être choisis parmi les négociants du Comité d'agriculture et de commerce qui n'avaient aucun intérêt économique dans les colonies. Son rapport du 12 novembre 1789 dépassa en fait le simple cadre commercial, pour évoquer le problème de l'esclavage ${ }^{16}$. Le texte ne fut pas prononcé mais il fit prendre conscience aux colons et négociants des ports du danger que représenterait la formation d'un comité où ils ne siègeraient pas.

8 Par ailleurs, l'activité des citoyens de couleur demandant à être admis comme députés à l'égal des Blancs, grâce au soutien de Grégoire, mettait également la stratégie de Massiac en danger. Un rapport avait été demandé au Comité de vérification pour examiner la légitimité de la demande ${ }^{17}$. Inquiété par la démarche, de Curt, député de Guadeloupe, décida de prendre les devants et réclama la création d'un comité spécifique, le 26 novembre 1789. Selon lui, les colonies "différ[aient] en tout de la métropole», il était nécessaire de donner aux « îles à sucre un régime particulier et des lois qui s'accord [ai]ent parfaitement avec leur position physique ». De Curt s'adressant à ses collègues poursuivait :

$$
\begin{aligned}
& \text { "vous avez formé des comités pour tous les objets soumis aux règles du calcul, ou } \\
& \text { qui, tenant à beaucoup de rapports, exigent les connaissances les plus étendues et } \\
& \text { des méditations profondes. Mais ces comités ne peuvent embrasser que l'intérieur } \\
& \text { du royaume; et si vous voulez organiser les colonies d'une manière qui vous assure } \\
& \text { à jamais les avantages de ces précieuses contrées, vous devez former un comité qui } \\
& \text { s'occupe sans délai d'en perfectionner les moyens }{ }^{18} \text {. }
\end{aligned}
$$

9 Or, les seuls experts légitimes sur les colonies, selon de Curt, étaient les négociants et colons qui devaient composer exclusivement le nouveau comité et préparer un plan de 
constitution spécifique aux colonies (en d'autres termes, conciliable avec l'esclavage, la traite négrière et l'exclusion politique des libres de couleur, même si l'orateur, comme ses collègues des colonies, évitait de prononcer ces mots).

Cependant la motion ne fut pas adoptée, notamment à cause de l'opposition du député de Nantes, Blin, membre du Club Massiac, encore fidèle à la stratégie du silence et craignant la mise en cause de l'Exclusif. La question fut de nouveau soulevée à plusieurs reprises entre le $1^{\mathrm{er}}$ et le 4 décembre 1789 : Malouet, grand propriétaire d'habitations réclamait la formation d'un comité dont les deux tiers seraient constitués de colons et de négociants ; Blin refusait parce que selon lui l'Assemblée n'avait pas les compétences pour établir une constitution qui devait être préparée dans les colonies elles-mêmes; enfin Grégoire s'y opposait également car il tenait à ce que le Comité de vérification ait préalablement établi l'admission des députés des libres de couleur ${ }^{19}$. Face à la violence des oppositions, la majorité de l'Assemblée qui se disait ignorante des réalités coloniales ne statua pas. Il fallut attendre l'aggravation des tensions coloniales et les nouvelles de Saint-Domingue et de Martinique pour que le Comité des colonies soit formé en mars 1790. Sa création traduisait un changement de stratégie radical de Massiac, soucieux d'éviter que les Amis des Noirs ne parvinssent à faire voter l'interdiction de la traite. Le Comité d'agriculture et de commerce avait tâché d'étouffer le débat ${ }^{20}$, mais Mirabeau, le $1^{\mathrm{er}}$ mars, avait présenté un projet d'abolition au club des Jacobins, qui, selon le Patriote français de Brissot, avait produit une forte impression ${ }^{21}$. Alexandre de Lameth, demanda donc le 2 mars 1790 la formation d'un comité spécifiquement chargé des affaires coloniales, qui trierait, classerait et synthétiserait les informations concernant les troubles antillais. Son frère, Charles, « comme Français ", " comme colon », précisa et étendit la mission que le Comité devait, selon lui, assumer : il lui faudrait élaborer une constitution spécifique aux colonies ${ }^{22}$. Les deux frères, en accord avec Barnave et le club Massiac, s'étaient ainsi coordonnés pour faire obstacle au débat sur la traite ${ }^{23}$. Alors que le traitement des questions coloniales avait été éparpillé entre plusieurs comités, tous les documents s'y rapportant seraient désormais centralisés dans un seul comité. En sous-main, la tactique visait à affaiblir Grégoire, président du Comité des rapports, et de ce fait contrôlant la circulation des informations coloniales.

\section{Un comité constituant, ministériel ou judiciaire?}

Bien que le Comité des colonies eût été chargé de concevoir une constitution coloniale, sa raison d'être fut mise en cause dès le premier projet de décret présenté par son rapporteur, Barnave, le 8 mars 1790. Ceux-là même qui avaient réclamé la formation du Comité, distinguant le régime intérieur du régime extérieur, entendaient accorder aux assemblées coloniales un pouvoir constituant indirect, sous réserve de la sanction de l'Assemblée nationale et de l'acceptation du roi $^{24}$. Le décret, voté sans discussions, stipulait que l'Assemblée nationale «n'avait jamais entendu comprendre [les colonies] dans la constitution». Les colonies elles-mêmes devaient émettre des vœux constitutionnels, compatibles «avec leurs convenances locales et particulières » et les « principes généraux qui lient les colonies à la métropole ». L'article 6 précisait en outre que l'Assemblée nationale « n'a[vait] entendu rien innover dans aucune des branches du commerce soit direct, soit indirect de la France avec ses colonies ; met[ait] les colons et leurs propriétés sous la sauvegarde spéciale de la nation $»^{25}$. Le débat sur la traite négrière ne put donc se tenir, la parole n'ayant pas été accordée aux Amis des Noirs. Quant aux 
réformes relatives au régime prohibitif et du ressort exclusif de l'Assemblée, elles étaient remises à plus tard, afin d'occulter les éternelles divisions entre négociants et planteurs. Le Comité était donc censé passer au second plan puisque les assemblées coloniales se voyaient attribuer, par son truchement, le rôle qui lui était initialement dévolu.

En réalité, ce projet ne résista pas à l'épreuve des faits, de telle sorte que le Comité se pérennisa tandis que ses compétences s'étendirent. Les crises politiques de Martinique et de Saint-Domingue annonçaient le début de guerres civiles : l'Assemblée nationale, pour éviter de "perdre les colonies", s'estima donc contrainte d'intervenir et le Comité des colonies devait jouer le premier rôle. Or, initialement, les documents relatifs aux troubles de Martinique étaient renvoyés au Comité des rapports, le Comité des colonies n'ayant pas pour vocation de veiller au maintien de l'ordre public. La captation de cette compétence s'avéra progressive et non institutionnalisée ${ }^{26}$. Il s'agissait désormais de centraliser toutes les affaires coloniales. Le Comité fut de surcroît chargé de démêler l'écheveau complexe des conflits antillais. Les rapports présentés par Barnave le 12 octobre et le 29 novembre 1790 devaient mettre en récit les troubles, dessiner les partis et examiner des informations parcellaires qu'on découvrait à Paris avec un mois et demi de retard - au mieux ${ }^{27}$. Le Comité se faisait donc l'interprète des évènements, le garant de l'intelligibilité du monde colonial $^{28}$. De la sorte, les membres du Comité entendaient contrôler les canaux de l'information, au point de prétendre monopoliser toute législation concernant les colonies. Dans cette optique, Moreau de Saint-Méry, député de Martinique et « expert » en droit colonial ${ }^{29}$, alla jusqu'à proposer, le 11 janvier $1791:$ « $1^{\circ}$ que les objets qui intéresseront immédiatement les colonies ne pourr[aie]nt lui être présentés que par son Comité colonial; $2^{\circ}$ que les autres comités ne pourr[aie]nt soumettre à sa délibération aucune disposition relative aux colonies, ni prendre aucun arrêté à cet égard, sans en avoir préalablement conféré avec le Comité colonial». Robespierre répliqua que la motion tendait à "gêner la liberté qu'[aurait] l'Assemblée de décréter, et chaque membre de proposer ce qui serait trouvé utile aux colonies », tandis que Pétion soulignait que la proposition tendrait à «rendre le Comité colonial très despotique $\aleph^{30}$. La proposition fut rejetée, mais témoignait d'une double ambition en partie déçue: accaparer la politique coloniale de l'Assemblée; assurer l'unité et la spécificité du droit colonial.

13 Cette aspiration traduisait néanmoins une extension pratique sinon institutionnelle des compétences du Comité - tant du point de vue législatif qu'exécutif. Les rapports de l'automne 1790 revenaient ainsi sur le partage entre métropole et colonies qui avait été établi, le 8 mars, et précisé dans l'instruction du 28 mars, puisqu'afin de «rassurer les colons ", le considérant du décret du 12 octobre stipulait que l'Assemblée nationale avait «la ferme volonté d'établir, comme article constitutionnel dans leur organisation, qu'aucunes lois sur l'état des personnes ne ser[aient] décrétées pour les colonies que sur la demande précise et formelle de leurs assemblées coloniales $»^{31}$. L'Assemblée nationale, en votant le texte du Comité, perdait son droit d'initiative sur l'esclavage. En outre, le 29 novembre, le Comité fut chargé - sur sa demande - d'adresser aux assemblées coloniales des instructions susceptibles d'éclairer leurs travaux constitutionnels, car les événements prouvaient selon Barnave que les colonies «manquaient de lumières». Pour pallier ces lacunes, surtout dans les petites colonies, l'Assemblée nationale, par l'intermédiaire du Comité, proposerait un modèle de constitution coloniale qui ne revêtirait cependant aucun caractère obligatoire : cette mission aboutit les 14-15 juin 1791 lorsque le décret accompagnant les instructions fut adoptés ${ }^{2}$. 
Parallèlement, le Comité s'immisçait progressivement sur le terrain de l'exécutif. En ce sens, le décret du 29 novembre établit un précédent extrêmement important en faisant désigner une commission civile chargée de rétablir l'ordre dans les Iles-du-Vent; le $1^{\mathrm{er}}$ février 1791, sur la proposition du Comité, des commissions analogues furent formées pour Saint-Domingue et la Guyane ${ }^{33}$. Dans ces deux décrets, le Comité priait le roi d'expédier des forces militaires aux Antilles, si bien qu'il en venait à coordonner l'action exécutive des différents comités de l'Assemblée et du ministère de la Marine ${ }^{34}$. A ce titre, le Comité prêtait le flanc aux critiques l'accusant de miner la séparation des pouvoirs, d'autant que le ministre-lui-même était désigné comme complice de ce détournement.

Le 20 janvier 1791, Monneron, député de Pondichéry, rapportant les troubles qui avaient bouleversé Chandernagor, demanda que le Comité des colonies se chargeât également des colonies orientales. Or, Barnave estimait que les compétences du Comité se limitaient implicitement à l'Amérique. Malouet rétorqua que la question coloniale devait être embrassée dans son ensemble car si l'on nommait « un comité asiatique, il faudrait aussi un comité africain, un comité amériquain, un comité pour chaque colonie ». La mission du Comité colonial consistait donc à "présenter les moyens de pacification et l'organisation de toutes les possessions lointaines; elles [devaient] être soumises aux mêmes lois et au même régime $\aleph^{35}$. Les attributions du Comité devenaient à proprement parler globales, tant du point de vue de la géographie que des prérogatives - législatives et exécutives. Pour embrasser le tout, définir une spécificité coloniale universelle et assurer la mise en œuvre d'un " principe métropolitain », le Comité adoptait une vocation impériale, par-delà les particularismes de telle ou telle colonie.

Ces fonctions globales furent conservées sous la Législative - sans être véritablement institutionnalisées. On vota la continuation du Comité le 12 octobre 1791, qui prit le nom plus général de «Comité des lois et règlements relatifs aux colonies ». A la différence des Comités militaire, de marine et diplomatique, la pérennisation du Comité colonial suscita peu de discussions. Dans l'opinion prononcée au club des Jacobins et publiée dans le Patriote français du 15 octobre 1791, Brissot lui-même expliquait qu'une « une assemblée ne p[ouvai]t pas elle-même rassembler des faits épars [...] Il [fallait ]donc un comité, ou tout au moins une commission spéciale pour cette classe d'objets; dans cette classe, [il fallait] ranger les colonies, trop éloignées de nous, pour que la vérité puisse aisément et constamment parvenir à un individu, trop agitées par les passions, pour qu'elle parvienne pure ». Si Brissot estimait que les colonies nécessitaient un comité spécifique, il mettait néanmoins en garde contre les dérives possibles de cette structure institutionnelle. C'est sans nul doute au Comité des colonies de la Constituante qu'il pensait, lorsqu'il condamna ceux qui mettaient leur statut de rapporteur au service de leur ambition personnelle, lorsqu'il mit en garde contre les relations incestueuses entre ministres et comités ou encore lorsqu'il dénonça la culture du secret qui «étouffe l'esprit public ${ }^{36}$. De fait, le Comité des colonies de la Législative perpétuait en mode mineur la logique de son prédécesseur : il se chargea surtout de rendre compte des " troubles de Saint-Domingue ", un défi encore plus difficile à relever depuis qu'avait débuté l'insurrection générale des esclaves dans la plaine du Nord, la nuit du 22 août 1791, qui fut connue à Paris fin octobre. Par ailleurs, à quelques exceptions près, il fut moins occupé à préparer des lois qu'à organiser la répression et à assurer les secours aux planteurs ${ }^{37}$. La principale loi adoptée par la deuxième Assemblée, celle du 24-28 mars/4 avril 1792 qui accordait des droits politiques aux libres de couleur, fut élaborée et votée contre la volonté du Comité - à 
l'instigation des députés Girondins. Lorsque la Législative se sépara, le Comité n'avait pas assumé les attributions qui avaient présidé à sa création.

Le Comité des colonies de la Convention, objet presque totalement oublié par l'historiographie, fut reconduit sous le titre encore plus général de "Comité colonial », le 2 octobre 1792. En réalité, il eut dans un premier temps pour principale attribution de veiller à l'exécution de la loi du 4 avril 1792, de contrôler les deux commissions envoyées dans les Antilles et d'en garantir le succès, soit en maintenant les commissaires en place, soit en les remplaçant ${ }^{38}$. Avec les arrivées successives des colons déportés de SaintDomingue par Sonthonax et Polverel, le Comité se transforma progressivement en tribunal chargé d'instruire le procès de la « Contre-Révolution » coloniale. Le décret du 17 décembre 1792 ordonnait que les individus débarqués devaient être mis en état d'arrestation et se présenter à la barre de l'Assemblée. Pour éviter l'engorgement face à l'afflux croissant des déportés, la Convention décréta le 24 décembre 1792 que les détenus prévenus d'avoir fomenté les troubles des colonies seraient préalablement entendus par les membres du Comité colonial, dont le rapport déciderait s'il y aurait lieu à accusation. L'occupation principale du Comité consista donc à mener les interrogatoires, seul parfois, mais le plus souvent réuni au Comité de sûreté générale ou au Comité de marine ${ }^{39}$. Parallèlement, le Comité devait présenter des rapports portant sur l'indemnisation des victimes et réfugiés de Saint-Domingue ${ }^{40}$. Accuser d'une part, secourir de l'autre: il s'agissait avant tout de classer les différentes catégories de colons entre Révolution et Contre-Révolution.

18 A partir de janvier 1793, le Comité devint peu à peu l'adjuvant du Comité de défense générale, où siégeaient trois de ses membres. Les projets des principaux décrets relatifs aux colonies furent en effet présentés au nom de ce grand comitée ${ }^{41}$. Le 5 mars, le Comité de marine et celui des colonies furent réorganisés et calqués sur le Ministère de la Marine et des Colonies (qui avait été lui-même remodelé le 14 février 1793) : il était ainsi précisé que le Comité des colonies correspondrait avec la $5^{\text {ème }}$ section du ministère. C'était engager une première étape vers la fusion des deux comités, laquelle fut effectivement arrêtée le 20 août 1793 par le Comité de salut public ${ }^{42}$, le Comité colonial étant du reste devenu une coquille vide depuis les journées des 31 mai/2 juin 1793 malgré le renouvellement de ses membres, le 6 juillet ${ }^{43}$. La réunion des deux comités en un seul ne fut toutefois officiellement décrétée par la Convention que le 7 fructidor an II (24 août 1794) : le Comité de marine et des colonies aurait désormais « la surveillance des objets attribués à la commission de marine et des colonies »; mais, strictement subordonné au Comité de salut public qui avait la charge de la défense des colonies, il devrait se « borner à la dénonciation des abus et à la proposition des lois relatives à cette partie ». Enfin, l'instruction du procès des différents protagonistes de l'«affaire des colonies», qui empoisonnait les travaux du Comité depuis le début de la Convention, fut externalisée un mois plus tard par la formation d'une commission spéciale, le 9 vendémiaire an III (30 septembre 1794$)^{44}$.

La guerre avait donc validé la position défendue sous la Constituante par Moreau de Saint-Méry, selon lequel la dissociation des deux départements n'avait aucun sens ${ }^{45}$. La Convention avait progressivement reproduit ce modèle ; et, de fait, le Comité de marine était devenu prépondérant dans les affaires coloniales à partir du mois d'avril 1793, du moins pour toutes les affaires dont le Comité de salut public ne s'était pas emparé. En dépit des attributions définies en 1790, le Comité s'avéra in fine faiblement impliqué dans la production législative, théorisant d'abord son incompétence, puis évitant tout 
simplement de faire la loi coloniale. Néanmoins, sa perpétuation signifiait le maintien d'un droit dérogatoire et avalisait une conception impériale. Le Comité était devenu un organe associé à l'exécutif, dans une logique de collaboration plutôt que de subordination; il s'était parallèlement transformé en une sorte de jury d'accusation colonial - deux attributions qui furent finalement dissociées. Mais sans doute les continuités sont-elles moins lisibles dans les compétences du Comité, que dans sa composition, marquée par la prépondérance des intérêts coloniaux.

\section{La composition du Comité : la prégnance de l' « intérêt colonial »}

Tout au long des débats sur la formation du Comité, la question de sa composition s'était révélée particulièrement sensible. Si l'Assemblée ne le réserva finalement pas aux seuls planteurs et négociants, nombre de ses membres officiels avaient en réalité des intérêts coloniaux, souvent économiques. Or, ce constat est valable pour l'ensemble des comités des trois Assemblées - l'exception de la Convention devant être nuancée, contrairement à l'image, moins formulée qu'implicite, du "comité anti-planteurs». Justifiant leur élection, la connaissance des spécificités locales ou du commerce colonial instituaient ces députés comme « experts».

Dans chacun des trois comités, le nombre des membres avait été initialement fixé à douze, auxquels étaient adjoints deux suppléants sous la Constituante, six sous la Législative et quatre sous la Convention. Le premier Comité ne fut pas renouvelé, mais des membres furent intégrés : Louis Monneron, le 20 janvier 1791, et six autres membres - précisément, comme nous le verrons, pour contrebalancer les «biais coloniaux » - le 22 août 1791. Le deuxième Comité conserva ses membres pendant toute la session, à l'exception de Vergniaud qui fut remplacé par le suppléant, Merlet, en mars 1792. Appliquant la règle du non-cumul, peu de membres élus au Comité colonial, le 11 octobre 1792, confirmèrent leur nomination. Les ténors des Amis des Noirs et/ou Girondins Grégoire, Kersaint et Garran-de-Coulon - déclinèrent, abandonnant Boyer-Fonfrède à un Comité plus modéré. Le 15 octobre 1792, malgré la promotion de suppléants, le Comité réorganisé ne comptait en réalité que neuf membres jusqu'à ce que Camboulas, Rouzet et Ferry fussent effectivement admis le 10 janvier 1793, puis Pomme, député de Cayenne, le 25 avril 1793. Au total, 39 députés (sans compter les 6 adjoints d'août 1791, et Despinassy étant seul à avoir siégé dans deux comités) siégèrent officiellement au sein du Comité.

Liste des membres du Comité des colonies

\begin{tabular}{|l|l|l|l|l|}
\hline Nom & $\begin{array}{l}\text { Mandat/ } \\
\text { département }\end{array}$ & Qualité/profession & $\begin{array}{l}\text { Entrée dans le } \\
\text { comité }\end{array}$ & $\begin{array}{l}\text { Connexion } \\
\text { coloniale }\end{array}$ \\
\hline \multicolumn{2}{|l|}{ Constituante } \\
\hline Alquier & La Rochelle & Avocat & $02 / 03 / 1790$ & Propriété \\
\hline Barnave & Dauphiné & Avocat & $02 / 03 / 1790$ & $\begin{array}{l}\text { Propriété } \\
\text { familiale }\end{array}$ \\
\hline
\end{tabular}




\begin{tabular}{|c|c|c|c|c|}
\hline Begouën & Caudebec & Négociant & $02 / 03 / 1790$ & $\begin{array}{l}\text { Propriété/ } \\
\text { négoce }\end{array}$ \\
\hline Champagny & Montbrison Lyon & Major (Marine) & $02 / 03 / 1790$ & $?$ \\
\hline Garesché & $\begin{array}{ll}\text { Saintes, } & \text { La } \\
\text { Rochelle } & \end{array}$ & Négociant & $02 / 03 / 1790$ & Négoce \\
\hline Gérard & Saint-Domingue & Planteur & $02 / 03 / 1790$ & Propriété \\
\hline Lameth & Péronne, Amiens & Militaire & $02 / 03 / 1790$ & $\begin{array}{l}\text { Propriété } \\
\text { familiale }\end{array}$ \\
\hline Le Chapelier & Rennes & Avocat & $02 / 03 / 1790$ & $?$ \\
\hline Monneron & Pondichéry & Négociant & $20 / 01 / 1791$ & Propriété \\
\hline $\begin{array}{ll}\text { Payen de } & \text { de } \\
\text { Boisneuf } & \end{array}$ & Tours & Habitant/militaire & $02 / 03 / 1790$ & $\begin{array}{l}\text { Propriété } \\
\text { familiale }\end{array}$ \\
\hline $\begin{array}{l}\text { Pèlerin de La } \\
\text { Buxière }\end{array}$ & Orléans & Médecin & $02 / 03 / 1790$ & Carrière \\
\hline $\begin{array}{l}\text { Reynaud de } \\
\text { Villeverd }\end{array}$ & Saint-Domingue & Habitant/militaire & $02 / 03 / 1790$ & Propriété \\
\hline Thouret & Rouen & Avocat & $02 / 03 / 1790$ & $?$ \\
\hline \multicolumn{5}{|l|}{ Législative } \\
\hline Barbotte & Orne & Avocat & $25 / 10 / 1791$ & $?$ \\
\hline Brulley & Indre-et-Loire & $\begin{array}{l}\text { Avocat du roi, trésorier } \\
\text { de France }\end{array}$ & $25 / 10 / 1791$ & $\begin{array}{l}\text { Propriété } \\
\text { familiale }\end{array}$ \\
\hline Courtin (aîné) & Seine-et-Oise & Négociant & $25 / 10 / 1791$ & $?$ \\
\hline Despinassy & Var & Militaire & $25 / 10 / 1791$ & Carrière \\
\hline Gonyn & Haute-Garonne & $\begin{array}{l}\text { Homme de loi/ } \\
\text { cultivateur }\end{array}$ & $25 / 10 / 1791$ & $?$ \\
\hline Journu-Aubert & Gironde & Négociant & $25 / 10 / 1791$ & Négoce \\
\hline Lecurel & Haute-Saône & Avocat & $25 / 10 / 1791$ & $?$ \\
\hline Levavasseur & Seine-Inférieure & Militaire & $25 / 10 / 1791$ & $\begin{array}{l}\text { Propriété } \\
\text { familiale }\end{array}$ \\
\hline Merlet & Maine-et-Loire & Homme de loi & $01 / 03 / 1792$ & $?$ \\
\hline Nogaret & Aveyron & Avocat & $25 / 10 / 1791$ & $?$ \\
\hline
\end{tabular}




\begin{tabular}{|c|c|c|c|c|}
\hline Queslin & Manche & Notaire & $25 / 10 / 1791$ & $?$ \\
\hline Tarbé & Seine-Inférieure & Négociant & $25 / 10 / 1791$ & Négoce \\
\hline Vergniaud & Gironde & Avocat & $25 / 10 / 1791$ & Familiale \\
\hline \multicolumn{5}{|l|}{ Convention* } \\
\hline Blanc & Marne & Commerçant & $11 / 10 / 1792$ & Carrière \\
\hline Boyer-Fonfrède & Gironde & Négociant & $11 / 10 / 1792$ & $\begin{array}{l}\text { Propriété/ } \\
\text { négoce }\end{array}$ \\
\hline Brunel & Hérault & Administrateur & $15 / 10 / 1792$ & Carrière \\
\hline Camboulas & Aveyron & Négociant & $10 / 01 / 1793$ & Familiale \\
\hline $\begin{array}{l}\text { Creuzé- } \\
\text { Latouche }\end{array}$ & Vienne & Avocat/homme de loi & $15 / 10 / 1792$ & $\begin{array}{l}\text { Propriété } \\
\text { familiale }\end{array}$ \\
\hline Despinassy & Var & Militaire & $10 / 01 / 1793$ & Carrière \\
\hline Ferry & Ardennes & Professeur & $10 / 01 / 1793$ & $?$ \\
\hline Guillermin & Saône-et-Loire & Militaire & $11 / 10 / 1792$ & $?$ \\
\hline Mazade-Percin & Haute Garonne & Homme de loi & $11 / 10 / 1792$ & Carrière \\
\hline $\begin{array}{l}\text { Pénières- } \\
\text { Delzors }\end{array}$ & Corrèze & Avocat et syndic & $15 / 10 / 1792$ & $?$ \\
\hline Pomme & Cayenne & $?$ & $25 / 04 / 1793$ & Propriété \\
\hline Peyre & Basses-Alpes & Négociant & $15 / 10 / 1793$ & Négoce \\
\hline Rouzet & Haute-Garonne & Homme de loi & $10 / 01 / 1793$ & $?$ \\
\hline Taveau & Calvados & Administrateur & $15 / 10 / 1793$ & $?$ \\
\hline
\end{tabular}

L'adjonction du 10 janvier 1793 n'est pas mentionnée dans les Archives parlementaires; mais elle apparaît dans les procès-verbaux du Comité. Le même jour, Brunel, Boyer-Fonfrède et Pénières furent élus commissaires au Comité de défense générale, mais continuèrent à assister à certaines séances du Comité. AN, D*XVI 5, fo. 23.

* L'adjonction du 10 janvier 1793 n'est pas mentionnée dans les Archives parlementaires; mais elle apparaît dans les procès-verbaux du Comité. Le même jour, Brunel, Boyer-Fonfrède et Pénières furent élus commissaires au Comité de défense générale, mais continuèrent à assister à certaines séances du Comité. AN, D*XVI 5, fo. 23.

22 A l'exclusion des six membres imposés au Comité en août 1791, les profils socioéconomiques des membres titulaires présentent de nombreuses analogies. Sous la Constituante, on compte quatre avocats, deux négociants, trois militaires et un «habitant»; mais c'est la prépondérance des propriétaires de plantations qu'il faut souligner. Au moins huit d'entre eux étaient en effet plus ou moins directement dans ce cas (par exemple, Alquier avait pour épouse une riche propriétaire). En outre, les non- 
propriétaires étaient liés au monde colonial par d'autres biais : Barnave, ami des Lameth, était également le parent de Bacon de la Chevalerie, l'un des planteurs les plus turbulents de Saint-Domingue ; Thouret et Le Chapelier étaient quant à eux issus de provinces dont les élites avaient fait fortune dans le commerce colonial. Sous la Législative, si les députés propriétaires d'habitations y étaient nettement moins nombreux que dans la précédente Assemblée, ils étaient en revanche surreprésentés dans le Comité. Outre les juristes et avocats (9 sur 13), figuraient trois négociants et deux militaires. Le monde du commerce, qui y était en force, avait des relations d'affaires avec les Antilles. Le père de Levavasseur comptait parmi les principaux négociants de Rouen. Vergniaud avait pour cousin un sénéchal de Saint-Domingue avec lequel il entretenait une correspondance suivie. Le beau-frère du Girondin, Boyer-Fonfrède, appartenait à une grande famille du négoce bordelais. Ancien capitaine à Saint-Domingue, Despinassy y avait peut-être des intérêts. Encore une fois, les départements maritimes étaient surreprésentés (quatre Normands et deux Girondins).

Le Comité de la Convention comptait moins de propriétaires d'habitation mais davantage d'individus qui avaient réellement vécu dans les colonies. Plusieurs y étaient nés ou y avaient assumé des fonctions administratives, au service du ministère de la Marine. Blanc, qui s'était enrichi dans le négoce du vin en Haute-Marne, était un créole de Martinique; Brunel avait été successivement sénéchal et lieutenant-général en Ile-deFrance avant de se fixer à Béziers. Quant à Mazade-Percin, il présentait une véritable carrière impériale : procureur du roi et sénéchal à Bourbon, il était devenu commissaire ordonnateur de la partie du Nord à Saint-Domingue en 1789. Alors que Boyer-Fonfrède représentait le grand commerce colonial et que Peyre avait essayé de percer en Martinique, les députés n'ayant aucun intérêt économique connu dans les Antilles (Ferry, Pénières, Taveau) y semblent à peine plus nombreux que dans les comités précédents. Les connexions pouvaient aussi être indirectes : Creuzé-Latouche avait, en la personne de son cousin, le député Creuzé-Pascal, une connexion avec Saint-Domingue ${ }^{46}$.

La coloration politique des comités est peut-être moins pertinente que celle de la proximité des membres avec les divers groupes de pression. En effet, c'étaient les "patriotes", avec Le Chapelier et Barnave, qui semblaient avoir la haute main sur le Comité, comme s'en était réjoui Brissot dans son journal. Or, la proximité du Comité avec la société des colons apparut progressivement. Bégouen était en effet lié au club Massiac par l'intermédiaire du grand négociant havrais Foäche, de même qu'Alexandre de Lameth par son frère Charles. Barnave lui-même était en relation avec de nombreux membres qu'il invita plusieurs fois à se réunir chez lui ${ }^{47}$. Cette proximité devint évidente au printemps 1791, lorsque le Comité soutint le décret du 13 mai stipulant qu'« aucune loi sur l'état des personnes non libres ne pourra[it] être faite par le corps législatif pour les Colonies, que sur la demande formelle et spontanée des assemblées » et lorsqu'il s'était opposé de toutes ses forces au décret du 15 mai accordant les droits politiques aux libres de couleur nés de père et mère libres. Pour contrer ces pressions et atténuer l'influence de Massiac, le 22 août 1791, Destutt de Tracy et Regnaud de Saint-Jean d'Angély réclamèrent l'adjonction de six nouveaux membres, étrangers au comité ${ }^{48}$. Cependant dès le 29 août 1791, quatre d'entre eux présentèrent une démission collective. Tracy le motivait par son opposition à « la persistance du comité dans un système qu[il avait] toujours combattu »; La Rochefoucauld ajoutait que le comité tâchait de contrecarrer le décret du 15 mai 1791, alors qu'il était censé assurer son application. Brostaret précisait : «j'ai cru devoir y rester pour appuyer l'opinion que je crois bonne et je ne me rebuterai 
pas en présence des difficultés que nous éprouvons, parce que je sais que les comités ne font pas la loi dans l'Assemblée nationale, qu'ils ne font que la lui proposer et que, quel que soit le décret proposé par la majorité du comité, il reste toujours à la minorité le droit de faire ses objections, ses propositions : l'Assemblée peut alors connaître qui a raison ». A la suite de ces déclarations, Regnaud demanda le renouvellement complet du Comité au nom de «l'intérêt national »- cette motion fut ajournée et finalement non adoptée ${ }^{49}$. Au lendemain du décret du 24 septembre 1791, abrogeant le décret du 15 mai, il y eut bien conjonction entre la scission feuillante (Barnave ayant été exclu des Jacobins à la suite du débat sur les colonies), la stratégie du club Massiac et la politique du Comité colonial ${ }^{50}$.

Sous la Législative, l'entrée de Vergniaud marqua l'admission d'un proche des Amis des Noirs et d'un jacobin avancé. Avec Ducos, il avait milité pour que des droits politiques fussent accordés aux libres de couleur ; mais, ce «noyau de la Gironde » avait fait fortune dans l'économie coloniale et l'esclavage : l'abolitionnisme devait être mis au service d'une réforme économique profonde. Cette dimension permet sans doute de mieux comprendre les accommodements de ces Girondins qui reportèrent l'abolition graduelle à une époque plus favorable. Du reste, Vergniaud n'était en aucun cas l'homme fort du Comité : face à lui, Journu-Aubert, appartenant au même milieu bordelais mais inscrit aux Feuillants, et surtout Charles Tarbé, frère du ministre des Contributions et grand négociant rouennais, dominaient le Comité de la Législative. Or, ces derniers étaient la tête de pont du Club Massiac $^{51}$. Sous la Convention, malgré le nombre de députés de tendance girondine, il existait une minorité importante de "modérés » sur les questions coloniales, autour de Creuzé-Latouche (président) et Mazade-Percin (secrétaire) ${ }^{52}$. La vraie rupture n'intervint que lors de la nomination de Boyer-Fonfrède au Comité de défense générale et de l'admission de Camboulas - le neveu de Raynal, nettement plus radical que son oncle et farouchement opposé aux lobbyistes des planteurs. Reste que la position de ces deux députés favorables à Sonthonax et Polverel ${ }^{53}$ ne faisait pas l'unanimité au sein du Comité comme le prouve la séance du 23 janvier 1793 : après " une longue délibération ", le Comité proposa en effet d'annuler la plupart des arrestations opérées par les commissaires civils en octobre $1792^{54}$. Creuzé-Latouche assortit l'élargissement d'une indemnisation des individus déportés « arbitrairement » le $1^{\text {er }}$ févier ${ }^{55}$.

Se contenter d'une analyse interne du Comité, considéré comme une entité hermétique et unitaire, est partiellement fallacieux. Tout d'abord, le Comité était très souvent réuni à d'autres comités. Dès le 3 mars 1790, le Comité d'agriculture et de commerce, qui avait déjà préparé un rapport sur la traite, nommait quatre membres pour assister le Comité colonial ${ }^{56}$. Jusqu'en mars 1791 , ce Comité déjà élargi avait la haute main sur les projets de décret présentés à l'Assemblée; mais, sur la demande tactique de Barnave qui voulait atténuer les préventions contre lui, la Constituante décréta le 5 avril 1791 que le Comité colonial se réunirait officiellement aux comités d'agriculture et de commerce, de marine et de constitution, pour préparer les instructions constitutionnelles des colonies ${ }^{57}$. De fait, tous les projets proposés à partir de cette date le sont au nom des Comités réunis ${ }^{58}$, qui ont tenu 22 séances communes du 27 avril 1791 au 26 septembre 1791. Cependant, il ne faudrait pas en déduire que le poids des colons en aurait été diminué puisqu'au sein de ces comités figurent entre autres le député de Guadeloupe, de Curt (Marine), l'ancien administrateur de Cayenne et Saint-Domingue, Malouet (Marine), le député de Martinique Moreau de Saint-Méry (Agriculture et Commerce), le grand propriétaire Delattre (Agriculture et Commerce) - tous membres du club Massiac ou du moins ayant assisté à certaines de ses séances. Defermond et Legendre, du Comité de marine, peu 
favorables aux vues du Comité colonial, se trouvèrent d'autant plus isolés que la plupart des autres membres des comités ne participèrent nullement aux séances. Pour avoir soutenu le décret du 15 mai 1791, et rédigé seul des instructions destinées à accompagner le décret, Dupont de Nemours du Comité d'agriculture et de commerce fut violemment pris à partie par les membres du Comité lors des quatre séances auxquelles il assista; Rabaut Saint-Étienne fut également très mal reçu le 26 mai $^{59}$. Lors de la législature suivante, le Comité colonial était souvent réuni à de nombreux autres comités (Marine, Commerce, Militaire, Secours, Diplomatique...), mais non de façon aussi pérenne que précédemment. Sous la Convention, le Comité se réunit au moins trois fois avec les Comités de commerce et de finances en octobre 1792 pour régler des problèmes liés aux finances coloniales, deux fois avec le Comité de sûreté générale en novembre. Les réunions avec le Comité de la marine furent extrêmement nombreuses jusqu'à juillet 1793 : il y eut au moins 17 séances communes. Or, si, à partir de janvier 1793, au sein du Comité colonial, Camboulas exprimait hautement ses vues radicales grâce au soutien du Comité de défense générale, plusieurs membres du Comité de la marine se montraient beaucoup plus sensibles aux arguments des lobbyistes planteurs ${ }^{60}$ : par ce biais, ces derniers purent exercer une influence qui ne s'était pas démentie dans les comités successifs.

Cette influence pouvait aller jusqu'à la participation effective aux séances du Comité. Audelà des réunions décrétées par l'Assemblée nationale, le Comité colonial accueillait en son sein nombre d'individus qui n'en avaient pas reçu le mandat. Les notes de Moreau de Saint-Méry consignant les propos tenus lors de 56 séances du Comité colonial de la Constituante montrent que les discussions étaient ouvertes à des constituants qui n'avaient pas été élus membres, tels que les députés des colonies - Moreau lui-même (bien avant que la réunion au Comité d'agriculture et de commerce ne fût ordonnée), Dillon (Martinique), de Curt, La Charrière (Guadeloupe), Gouy d'Arsy, Reynaud, Villeblanche (Saint-Domingue), etc. Mais participèrent également aux séances plusieurs des « 85 », ceux des anciens membres de l'assemblée autonomiste de Saint-Domingue qui avaient débarqué à Brest, le 14 septembre 1790, et dont les menées avaient pourtant été condamnées par l'Assemblée sur le rapport de Barnave le 12 octobre $^{61}$. Le principal porteparole de l'ancienne assemblée coloniale n'était autre que Valentin de Cullion, qui avait ordonné la décapitation du sénéchal du Petit-Goâve, Ferrand de Baudières, pour avoir rédigé une pétition en faveur des libres de couleur en novembre $1789^{62}$. Participèrent également les députés de l'Assemblée du Nord de Saint-Domingue qui étaient venus en métropole dénoncer l'assemblée de Saint-Marc, tel Trémondrie, ou encore les députés extraordinaires de manufactures et de commerce, Rostagny et Abeille. Si ces individus ne pouvaient voter, ils participaient pleinement aux délibérations et monopolisèrent les discussions. Barnave comme Moreau de Saint-Méry parurent à toutes les séances ; Dillon, Cullion et Gouy y intervinrent quasiment à chaque fois, tandis que les membres officiels, Le Chapelier et Thouret, ne se présentèrent qu'une seule fois.

Sous l'Assemblée législative, nous ne disposons que de procès-verbaux officiels, d'une extrême discrétion quant à la participation effective des membres et à l'intervention éventuelle d'éléments extérieurs. Si le proche des Girondins, Kersaint, fut autorisé à présenter un rapport sur le «rétablissement de l'ordre dans les colonies », les 10-11 novembre $1791^{63}$, ce furent bien davantage les lobbyistes des colons blancs qui avaient l'oreille du Comité. Les registres de la commission de Saint-Domingue, mandatée par l'assemblée coloniale pour défendre les intérêts des planteurs, en témoignent 
exemplairement. Ainsi annoncent-ils, le 6 décembre 1791 : « Déjà nous avons vu tous les membres du Comité Colonial, nous continuerons à les voir collectivement et individuellement, nous assisterons à leurs séances et ne négligerons aucuns des moyens qui pourront contribuer au succès de notre affaire»; le 16 décembre: «Le comité colonial que nous voions souvent nous paroit être dans les meilleures intentions; il avait besoin de lumières et de documents : nous nous sommes hâtés de lui participer tous ceux qui étoient en notre pouvoir ». Ces bonnes relations avec le Comité ne furent pas même affectées par le 10 août 1792 puisque la commission, le 25 août, " propos[ait] de faire remerciements tant au comité colonial qu'au ministre de la Marine sur ce que prenant en considération les observations de la commission ils [avaient] fait accompagner l'envoi à St. Domingue du décret de liberté et d'égalité d'instructions qui $p$ [ouvaient] prévenir les suites funestes qu'aurait eu la promulgation pure et simple de ce décret $»^{64}$. Sous la Convention, les principaux porte-parole de la commission, Page et Brulley réussirent à se faire entendre du Comité de défense générale et se rendirent à plusieurs reprises aux séances du Comité colonial ${ }^{65}$. Le 8 février, par exemple, ils "entretinrent le comité d'affaires de leurs colonies et [donnèrent] des renseignements fâcheux sur la conduite du commissaire civil Sonthonax $»^{66}$. Les 25-26 mars et 15-17 mai 1793, devant les membres des Comités des colonies et de marine réunis, ils furent confrontés à Julien Raymond: de ce face-à-face dépendrait le sort du décret du 5 mars, qui avalisait la formation de régiments constitués de libres, et qui autorisait les commissaires à faire dans «les règlements de discipline et de police des ateliers, tous les changements qu'ils juger[aient] nécessaires ${ }^{67}$. On comprendra donc que s'en tenir à la composition officielle ne permet pas de comprendre le fonctionnement du Comité, dont les frontières avec les groupes de pression s'avérèrent éminemment poreuses.

\section{« Nous sommes ici en famille » : des débats coloniaux sous le sceau du secret}

Pour comprendre le fonctionnement du Comité, il faut apprendre à lire entre les lignes, tant les procès-verbaux officiels sont elliptiques. C'est pourquoi les notes détaillées de Moreau de Saint-Méry constituent un document exceptionnel, les propos des intervenants y étant cités précisément. Pourtant, ces pages n'auraient pas dû nous parvenir, puisque Moreau avait été prié de supprimer les notes et « le fai[sai]t depuis le commencement $d u$ travail des instructions $»^{68}$. Les procès-verbaux du Comité sous la Législative manifestent bien davantage de discrétion, malgré les deux secrétaires-commis affectés. Alors que le mois de décembre 1791 fut marqué par le débat sur l'insurrection générale de Saint-Domingue et par un affrontement très violent entre Tarbé et Brissot sur les droits des libres de couleur, on lit que « les délibérations du comité n'ayant porté sur aucun objet principal pendant le mois de décembre, on a[vait] cru pouvoir se dispenser de les écrire ${ }^{69}$. Le secrétaire-commis Salley, qui rédige les procès-verbaux du Comité colonial de la Convention ${ }^{70}$, sait également manier la litote et l'euphémisme. Ainsi-écritil : «En suite du décret rendu par la Convention [le 5 mars 1793], les colons des isles du vent et des isles sous le vent ont été entendus et les Comités réunis de marine et des colonies les ont ajournés après une légère discussion à demain 7 heures du soir $»^{71}$. Les commissaires Page et Brulley rapportent quant à eux une version nettement moins édulcorée de cette «légère discussion »: Camboulas se serait en fait écrié qu' «il étoit ridicule de prolonger la discussion sur ce décret ; puisqu'il en étoit ainsi, il falloit discuter 
la grande question de savoir s'il étoit important pour la France d'avoir des colonies, \& si elle pouvoit y laisser exister la servitude. Cette phrase, dictée par l'impatience, a excité une rumeur générale dans le comité ; on s'est écrié que ce n'étoit pas l'instant d'agiter cette question; alors le citoyen Camboulas a dit qu'il retiroit sa proposition; qu'il ne l'avoit faite, que pour prouver qu'il ne falloit pas remonter au principe, parce que IN PRINCIPIUM ERAT VERBUM $»^{72}$. Cependant, comme l'avait dit Barnave, ces échanges devaient rester " en famille ", même si des fuites étaient toujours à prévoir ${ }^{73}$.

Compte-tenu du biais des sources, il apparaît dès lors hasardeux de rendre compte de la régularité des séances ou de leur fréquence. Sous la Constituante, 56 séances sont attestées; mais on ignore totalement ce qu'il en est pour 1790. Le Comité de la Législative se réunit au moins 11 fois en novembre 1791 ; puis aucune séance n'est mentionnée jusqu'en février 1792 ; et on relève 14 séances seulement jusqu'à la fin de la session. Quant au Comité de la Convention, il se serait réuni 59 fois entre le 27 octobre 1792 et le 27 avril $1793^{74}$. A ces séances, il faut en ajouter 12 autres qui eurent lieu dans le local du Comité de marine et auxquelles intervinrent plusieurs membres du Comité colonial. Quant à la participation effective des membres aux séances, elle n'est pas toujours évidente. Sous la Constituante, les ténors de l'Assemblée tels que Lameth, Le Chapelier et Thouret furent chacun membres de plus de quatre comités différents. Thouret ne se serait présenté la première fois au Comité que le 9 octobre $1790^{75}$ ! Sous la Législative, l'assiduité des membres semblait également très inégale: seuls neuf d'entre eux siégeaient effectivement lors de la première réunion. Bien que Vergniaud eût été élu président, il se signala par son absence, laissant le champ libre à Tarbé. Ainsi le député de Gironde déclara-t-il à l'Assemblée qu'il ignorait une partie du rapport présenté le 11 janvier 1792, dont il se désolidarisait complètement. Tarbé répliqua alors : « $\mathrm{M}$. Vergniaud ne vient point aussi souvent aux séances du comité qu'il le désirerait $\aleph^{76}$. Plusieurs membres qui n'intervinrent jamais dans l'hémicycle ne s'impliquèrent pas davantage dans le Comité (Barbotte, Courtin). Enfin, certains conventionnels s'absentèrent longuement, ayant été envoyés en mission dans les départements, tel Mazade-Percin à Rochefort entre janvier et juillet 1793.

31 Ces éléments nous portent à nous interroger sur le caractère collectif des activités du Comité, dont la tâche principale consistait à préparer des rapports et des projets de décret. La distribution de ces travaux était donc cruciale, car le membre désigné se devait de rassembler les informations, les trier et les hiérarchiser, avant de soumettre son interprétation et ses solutions législatives ou exécutives au reste du Comité. A ce titre, la place de rapporteur s'avérait bien plus importante que celle de président ou de secrétaire, comme le prouve la marginalisation de Vergniaud dans le Comité sous la Législative. Le sujet était en fait brûlant : ainsi Linguet accusa-t-il Barnave d'avoir troqué son statut de rapporteur pour celui de "ministre des colonies ». Ce dernier aurait mené ses travaux à Massiac et non au sein d'un comité trompe-l'œil. Si l'absence de procès-verbaux ne permet pas de déterminer la participation du Comité à l'élaboration des textes, on sait qu'il consulta personnellement la Garde Nationale de Bordeaux, ainsi que Billard, membre influent du Club Massiac ${ }^{77}$. Sous la Législative, la préparation des rapports était répartie entre les différents membres, sur des critères de spécialisation géographique ${ }^{78}$. Mais les rapports les plus importants - car ils déterminaient toute la politique coloniale de l'Assemblée -, ceux qui portaient sur Saint-Domingue, revenaient à Tarbé et à JournuAuber, les proches de Massiac ${ }^{79}$. Les autres membres étaient souvent réduits à produire des textes sur des sujets mineurs et diverses pétitions à traiter concernant des cas 
particuliers. Il ne faudrait toutefois pas exagérer le cloisonnement des travaux, ni occulter les marges importantes de débat. Sous la Législative, par exemple, le choix de lettres à lire en séance avait donné lieu à « de longues discussions », le 9 novembre $1791^{80}$, ou, sous la Convention, la lecture du rapport Camboulas fit l'objet de plusieurs relectures, les 4 et 8 février $1793^{81}$.

Nos sources ne nous permettent pas d'approfondir l'analyse pour ces deux comités; et sans doute les modalités de fonctionnement variaient-elles selon les circonstances. En revanche, les notes de Moreau prouvent qu'il y eut bien un débat au sein du Comité colonial élargi entre octobre 1790 et juin 1791. L'historiographie a eu tendance à réduire ce Comité au rôle de garant du "système colonial ", dans une perspective sans doute trop monolithique. Ce qui frappe au contraire, c'est la violence des altercations, Barnave n'écrasant pas les discussions, tant s'en faut. Lorsque Dillon proposa de déclarer citoyens actifs les libres de couleur, Cullion défia le député de Martinique en ces termes : «avant cela il faut que vous tuiés quelques-uns de nous. Moi par exemple, je le déclare ${ }^{82}$. La préparation des instructions constitutionnelles mit en effet au jour une grande variété de désaccords autour de l'autonomie politique des colonies, de la représentation coloniale à l'Assemblée nationale, de l'existence d'un tribunal de cassation, de la création d'un ministère spécifique aux colonies, du découpage administratif de Saint-Domingue, des contributions exigibles d'un citoyen actif, de l'unification du droit colonial, du régime prohibitif ou encore des droits à accorder aux libres de couleur... Si Barnave entendait favoriser "un principe métropolitain", en assurant la souveraineté nationale par la représentation coloniale et le maintien d'un Exclusif favorable au commerce de métropole, Le Chapelier ne voulait accorder qu'un droit de pétition à des assemblées coloniales, tandis que Gouy demandait que les colonies fussent assimilées à des départements...

Surtout le Comité servait à rendre possibles des débats qui eussent été inadmissibles dans l'hémicycle ou qui eussent pu tourner en faveur des " philanthropes ». La définition de la citoyenneté active, qui supposait une comparaison serrée avec les choix opérés par l'Assemblée nationale pour la métropole, est en ce sens un exemple particulièrement éclairant dans la mesure où se superposent les considérations juridiques, sociales et de couleur. Pour Barnave, il était essentiel d'« intéresser tous les Blancs", " sous condition d'une contribution directe parce qu'elle suppos[ait] une propriété au moins en esclaves et que le maître [était] intéressé au maintien de l'ordre »: il fallait donc établir une citoyenneté active fondée sur la propriété d'esclaves et une domiciliation de trois ans. Trouvant ces critères trop laxistes, Malouet s'en insurgea parce que selon lui, il était nécessaire d'exclure de la chose publique les petits blancs - commis, économes, souséconomes - outre ces «aventuriers » achetables, qui, craignait Dillon, pouvaient affluer par millions dans les colonies. "Comment Mrs les députés [avaien]t-ils pu consentir à n'exiger qu'une propriété de 15 mille livres pour l'éligibilité à St. Domingue », demanda Malouet : « qui n'a pas cela ? »; le bien peu fortuné Moreau de Saint-Méry répondit : « il y a chés moi des hommes pauvres et plein de mérite ». Pour Reynaud, afin de « conserver la dignité de la couleur, [il fallait] repousser les petits blancs car les esclaves les mépris [ai]ent » : le débat portait donc aussi sur les droits à accorder aux libres. Devaient-ils être considérés comme des alliés en tant que propriétaires d'esclaves ou au contraire constituer cette classe intermédiaire qui préserverait le préjugé de couleur? Malouet estimait ainsi qu' "ils étaient le rempart le plus sûr contre les ennemis intérieurs ", alors que La Galissonnière, rejetant les comparaisons avec Sparte et Athènes, affirmait 
hautement "qu'il fallait conserver aux Colonies l'aristocratie de la couleur $»^{83}$. Tout le répertoire du colonial était donc exploré, dans une langue du privilège inacceptable à l'Assemblée ; mais en ce lieu ne faisait consensus qu'un seul principe : la préservation de l'esclavage.

\section{Maîtriser le vote du projet et l'exécution de la loi : l'emprise sur l'Assemblée et le ministère}

Espace de débats coloniaux, le Comité était également l'officine où se préparaient les séances de l'Assemblée. Ses membres avaient d'abord le souci de contrôler les informations coloniales: Barnave fit en sorte que tous les documents portant sur les colonies fussent examinés au préalable par le Comité, au nom de l'expertise des planteurs et négociants. Le 29 juillet 1790, lorsqu'un courrier de l'assemblée coloniale de SaintDomingue parvint à l'Assemblée nationale, il en empêcha la lecture et demanda à ce qu'il fût d'abord examiné par le Comité afin de ne pas créer la panique ${ }^{84}$. Le tri et le choix des pièces présentées faisaient l'objet d'un travail minutieux comme en témoignent les brouillons du projet visant à abroger le décret du 15 mai $1791^{85}$. Quand le Comité parvint à ses fins le 24 septembre, les documents justificatifs avaient été soigneusement sélectionnés ( 40 hostiles au décret, 8 favorables seulement), suscitant l'ire de Reubel qui déclarait avoir « entendu la lecture de différentes adresses qui étaient sorties du même moule, préparées d'avance et déjà faites et signées avant que le décret fût rendu ${ }^{86}$. La pratique était coutumière : en février 1791, Barnave avait déclaré qu'« il [fallait] qu'il vienne des députés de Bordeaux, des autres ports \& qui dans une adresse modérée, ferme, qui dise que l'intérêt des colonies, de la métropole est qu'on cesse de les agiter "; Trémondrie expliquait qu'il était indispensable d'« écrire aux chambres de commerce, aux Assemblées générales de commerce »; " comme on a fait sur le décret du 8 mars ", précisait Reynaud. Et Moreau de Saint-Méry d'écrire une lettre destinée à toutes les Chambres de commerce ${ }^{87}$, afin de diffuser un argumentaire clef-en-main - ce que la langue médiatico-politique appellerait aujourd'hui des « éléments de langage ». Le Comité ne se contentait donc pas de filtrer les informations, il les produisait. Ces manœuvres étaient mises au service d'une cause : constitutionnaliser l'initiative des colonies sur l'état des personnes de manière à contrecarrer les Amis des Noirs, qui s'annonçaient comme les futurs hommes forts de la législature suivante.

Cependant, l'expérience de la Constituante informa les pratiques des acteurs sous la Législative. Pour éviter ces écueils, Tarbé adopta une stratégie dilatoire, consistant à tâcher de "faire oublier" les colonies. Les commissaires notèrent à ce sujet «que l'intention du Comité colonial étoit d'attendre l'effet qu'aura[it] produit à St. Domingue la connaissance de la loi du 24 septembre $»^{88}$. Les nouvelles de l'insurrection générale parvenant régulièrement à l'Assemblée, cette tactique s'avéra impraticable et le Comité fut tenu de présenter un rapport. Le 30 novembre 1791, des colons firent le récit des "massacres de Saint-Domingue »; mais le lendemain, Tarbé annonçait que le Comité colonial n'était pas en mesure de présenter son rapport et demandait que la lecture n'en fût faite que le 10. Brissot en profita pour prendre l'initiative et prononcer un très long discours sur Saint-Domingue ${ }^{89}$. Il s'agissait de couper l'herbe sous le pied du Comité, avec l'aide probable de Vergniaud, de manière à fixer les termes du débat sans laisser le champ libre à Tarbé. Minoritaires dans le Comité, les « Brissotins " parvinrent ainsi à faire voter le décret du 7 décembre, qui organisait la répression et justifiait les concordats, au terme 
d'une séance houleuse avant la lecture du rapport du Comitép ${ }^{90}$. L'influence des Girondins ne cessant de croître tout au long de la session, le Comité tenta en février 1792 de brouiller les cartes en confiant la lecture de son rapport à Journu-Auber, lui-même député de la Gironde, dont " l'influence promettait plus de succès $~^{91}$. Toutefois, ces stratagèmes n'empêchèrent nullement le vote du décret du 28 mars 1792, car le Comité «joui[ssait] d'une assez grande défaveur dans l'assemblée ${ }^{29}$.

Mais le Comité n'exerçait pas seulement son influence sur l'Assemblée, il hantait également les couloirs du ministère de la Marine et des Colonies. Sous la Constituante et la Législative, le Comité colonial et le ministre avaient en effet collaboré dans la fabrique et dans l'(in-)exécution des lois (selon qu'elles étaient conformes aux vues du Comité). En amont, le ministre jouait un grand rôle dans le déclenchement du processus législatif : c'est lui qui suscitait les débats à l'Assemblée, en transmettant les informations concernant les colonies, notamment les lettres qu'il recevait des gouverneurs. Or, ces annonces étaient coordonnées avec le Comité. Ce fut par exemple le cas, le 22 août 1791, lorsque les nouvelles catastrophiques du gouverneur Blanchelande permirent d'enclencher la riposte législative aboutissant au décret du 24 septembre. En aval, la collaboration alla jusqu'à empêcher l'expédition des décrets non conformes aux vues du Comité, en particulier celui du 15 mai $1791^{93}$. Cette connivence attira les soupçons : le député Albitte déclara en séance, le 13 janvier 1792, après lecture d'un rapport de JournuAuber : « je suis étonné qu'on nous renvoie sans cesse au ministre pour avoir des avis et des plans. C'est de fait donner l'initiative au ministre sur tous les points qui concernent l'utilité publique $»^{94}$. Le Comité des colonies, à la différence du Comité de la marine, ne prit pas part à la campagne de dénonciations menée contre le ministre Bertrand de Molleville. Le successeur, Lacoste, était également fort apprécié des commissaires de Saint-Domingue, car "toute la confiance qu'il inspir[ait] à l'assemblée nationale ser [aient] emploiés pour la réussite de [leur] affaire». Sous la Convention, si le nouveau ministre, Monge, s'intéressa peu aux colonies, Page et Brulley trouvèrent en Adet, le chef du Bureau des Colonies, une oreille attentive, un fonctionnaire soucieux de défendre leurs intérêts ${ }^{95}$.

Lorsque l'esclavage fut aboli, le 16 pluviôse an II, l'Assemblée nationale n'avait plus en son sein de Comité colonial spécifique. Il serait sans doute hasardeux d'en induire une relation de cause à effet, mais l'existence même de ce Comité plaidait en faveur du pluralisme juridique. «Colonial»: sa seule dénomination offrait un argument contre l'assimilation. A n'en pas douter, il serait également faux de confondre le Comité de la Constituante et celui de la Convention, lequel défendit vigoureusement les droits des libres de couleur. Cependant, dans les trois comités, certaines constantes ne se démentirent pas: le profil «colonial» des membres, une semblable difficulté à interpréter les événements des Antilles ou des Mascareignes, le poids des lobbies considérés comme des sources d'informations - et surtout une conception impériale qui tâchait d'articuler une vue d'ensemble des colonies, visant ainsi à l'unification d'un droit colonial par-delà les particularismes locaux. En ce sens, le Comité s'inscrivait dans la filiation des commissions de législation instituées par les ministres Choiseul, Sartine et Castries, pour ordonner l'État impérial depuis la fin de la guerre de Sept ans ${ }^{96}$. Ces éléments de continuité, enchâssés dans une chronologie plus large, incitent à nuancer 
fortement l'opposition entre un comité «feuillant » et un comité " girondin », selon une grille de lecture masquant des dynamiques coloniales qui préexistent à 1789 .

Mais, outre cette spécificité coloniale, le Comité exacerbe en quelque sorte les tensions inhérentes au système des comités, car, en définitive, de quoi l'expertise coloniale étaitelle le nom? d'une approche technicienne du bien commun, du souci d'assurer la conservation de l'État impérial ou d'intérêts particuliers savamment maquillés ? Quand des individus non élus en viennent à rédiger des projets de loi ; quand on distribue les rôles à tenir dans l'hémicycle; quand les scenarii des séances sont écrits à l'avance, et que chacun tâche de jouer sa partition; qui, dès lors, parle en tribune? qu'est-ce que la représentation nationale?

\section{NOTES}

1. Jacques-Pierre BRISSOT DE WARVILLE, Lettre de J.-P. Brissot à M. Barnave sur ses rapports concernant les colonies, les décrets qui les ont suivis, leurs conséquences fatales..., Paris, Desenne, 1790, préface.

2. Annales politiques et littéraires, t. XVIII, $\mathrm{n}^{\circ} 158, \mathrm{p} .117$.

3. L'Ami des patriotes ou le défenseur de la Révolution, t. III, n 54, p. 389.

4. L'Ami des patriotes ou le défenseur de la Révolution, t. II, nº 25, p. 266.

5. Paul Cheney a judicieusement souligné l'ambivalence historiographique de Barnave: il fut considéré comme l'interprète de l'ascension de la bourgeoisie par l'école classique et le héros du tragique révolutionnaire par les historiens révisionnistes. Voir Paul CHENEY, Revolutionary Commerce: Globalization and the French Monarchy, Cambridge, Harvard University Press, 2010, p. 198-201.

6. Ibid., Souad DEGACHI, Barnave, rapporteur du comité des colonies, Révolution Française.net, 2007.

7. L'ouvrage de référence reste : Gabriel DEBIEN, Les colons de Saint-Domingue et la Révolution; essai sur le club Massiac (août 1789-Août 1792), Paris, A. Colin, 1953; voir aussi Déborah LIÉBART, «Un groupe de pression contre-révolutionnaire: le club Massiac sous la Constituante ", Annales historiques de la Révolution française, $\mathrm{n}^{\circ}$ 354, décembre 2008, p. 29-50.

8. Le seul travail d'ensemble est une thèse américaine, très difficile d'accès en France : Valerie Yow QUINNEY, The Committee on Colonies of the French Constituent Assembly, University of Wisconsin, 1967. Deux articles du même auteur en reprennent certains éléments: Valerie QUINNEY, «Decisions on Slavery, the Slave-Trade and Civil Rights for Negroes in the Early French Revolution ", The Journal of Negro History, vol. 55, n² 2, avril 1970, p. 544-557; Valerie QUINNEY, "The Problem of Civil Rights for Free Men of Color in the Early French Revolution », French Historical Studies, vol. 7, $\mathrm{n}^{\circ}$ 4, octobre 1972, p. 117-130.

9. Pour des raisons évidentes, l'historiographie, depuis très longtemps et jusqu'à récemment, s'est concentrée sur deux moments-clefs : la décision de ne pas appliquer les droits de l'homme dans les colonies sous la Constituante, et l'abolition de l'esclavage le 16 pluviôse an II. Voir par exemple sur la Constituante : Léon DESCHAMPS, Les Colonies pendant la Révolution. La Constituante et la réforme coloniale, Paris, Perrin, 1898; Henri JOUCLA, Le conseil supérieur des colonies et ses antécédents : avec de nombreux documents inédits et notamment les procès-verbaux du comité colonial de l'assemblée constituante, Paris, éd. du monde moderne, 1927 ; David GEGGUS, «Racial Equality, Slavery, and Colonial Secession during the Constituent Assembly », The American Historical Review, 
vol. 94, n 5, décembre 1989, p. 1290-1308; sur l'abolition : Yves Bénot, « Comment la Convention a-t-elle voté l'abolition de l'esclavage en l'an II », AHRF, n² 293-294, p. 349-361 ; Florence GAUTHIER, "Le rôle de la députation de Saint-Domingue dans l'abolition de l'esclavage» dans Marcel DORIGNY (dir.), Les abolitions de l'esclavage. De L.F. Sonthonax à V. Schoelcher, 1793,1794, 1848, Paris, Presses universitaires de Vincennes, UNESCO, 1995, p. 199-211; Jeremy D POPKIN, You Are All Free: the Haitian Revolution and the Abolition of Slavery, Cambridge, Cambridge University Press, 2010.

10. Parmi les travaux soulignant la rupture républicaine: Bernard GAINOT, «La Constitutionnalisation de la liberté générale sous le Directoire» dans M. Dorigny (éd.), op. cit., p. 213-229 ; Jean-Daniel PIQUET, L'émancipation des Noirs dans la Révolution française : 1789-1795, Paris, Karthala, 2002 ; ceux qui insistent sur le dualisme et minimisent la rupture : Josep M. FRADERA, «L'esclavage et la logique constitutionnelle des empires", Annales. Histoire, Sciences Sociales, vol. 63, $\mathrm{n}^{\circ} 3$, juin 2008, p. 533-560; Miranda F. SPIELER, "The Legal Structure of Colonial Rule during the French Revolution », The William and Mary Quarterly, vol. 66, n² 2, avril 2009, p. 365-408. 11. Le livre-tournant fut Yves BENOT, La Révolution française et la fin des colonies, Paris, la Découverte, 1988. Nous suivons également les analyses de Jean-Frédéric Schaub, invitant à ne pas séparer l'histoire coloniale de l'histoire générale : Jean-Frédéric SCHAUB, «La catégorie «études coloniales » est-elle indispensable? ", Annales HSS, n 3, 2008, p. 625-648.

12. Les enjeux de la formation du Comité sont présentés de façon très détaillée dans V. QUINNEY, op. cit., 1967, p. 24-166 : sauf mention spéciale, cette partie se fonde sur ces chapitres.

13. Blanche MAUREL, Saint-Domingue et la Révolution française. La représentation des colons en France de 1789 à 1795, Paris, PUF, 1943, p. 1-5 ; Malick GHACHEM, «The Trap of Representation : Sovereignty, Slavery and the Road to the Haitian Revolution ", Historical Reflections/Réflexions historiques, 20, $\mathrm{n}$ ${ }^{\circ} 1,2003$, p. 123-144. Furent successivement admis les députés de Guadeloupe (22 septembre 1789), de Martinique (14 octobre 1789), des comptoirs de l'Inde (19 septembre 1790), d'Ile-deFrance (12 février 1791) - soit un total de 17 députés et 26 suppléants.

14. Tous ces groupes ont fait l'objet de travaux spécifiques, parfois anciens : Joseph LETAConNoux, Le Comité des députés extraordinaires des manufactures et du commerce de France et l'œuvre économique de l'Assemblée constituante, 1789-1791, Paris, E. Leroux, 1913 ; Gabriel DEBIEN, op. cit., 1953 ; Florence GAUTHIER, L'aristocratie de l'épiderme : le combat de la Société des citoyens de couleur, 1789-1791, Paris, CNRS éd., 2007 ; Marcel DORIGNY et Bernard GAINOT, La Société des amis des Noirs, 1788-1799, Paris, EDICEF, 1998.

15. Archives parlementaires (AP), t. VIII, p. 554

16. Louis-Charles Gillet de La Jaqueminière, Rapport fait au nom de la section du comité d'agriculture et de commerce, chargée par l'Assemblée nationale de l'examen de la déclaration des députés de Saint-Domingue, relative à l'approvisionnement de l'île, Paris, Baudouin, 1789.

17. F. GAUTHIER, op. cit., p. 65.

18. AP, t. X, p. 263.

19. Ibid., p. 347-353.

20. F. GAUTHIER, op. cit., p. 63-65.

21. Patriote français, $\mathrm{n}^{\circ} 206,2$ mars 1790, p. 1. Or, dans ce texte publié par M. Dorigny, Mirabeau préconisait la création d'un comité pour "préparer la liberté ». Honoré-Gabriel de Riquetti MIRABEAU (comte de), Victor HUGo, Marcel DORIGNY et Société française d'étude du XVIIIe sIÈcLE, Les bières flottantes des négriers, Université de Saint-Etienne, 1999.

22. AP, t. XII, p. 6.

23. L'opinion de Pétion, non prononcée, fut néanmoins imprimée. Voir AP, t. XII, p. 78-92.

24. L'Assemblée nationale seule disposait toutefois du pouvoir constituant, puisqu'elle seule représentait la nation. L'article 4 stipulait ainsi : «Les plans, préparés dans lesdites assemblées coloniales, seront soumis à l'Assemblée nationale, pour être examinés, décrétés par elle, et présentés à l'acceptation et à la sanction du roi ». Ibid., p. 72. Par la suite, lors de plusieurs 
séances du Comité, Barnave rappela « la suprématie du corps national » et s'opposa vertement à l'idée qu'une section de l'empire pût être souveraine. ANOM, 87 MIOM 95, fo. 205, 250.

25. Ibid.

26. Lorsqu'Anthoine, au nom du Comité des rapports, présenta un projet de décret sur une affaire anecdotique de Martinique, le député de Bordeaux et membre de Massiac, Nairac, demanda à ce que les documents fussent renvoyés au Comité des colonies préalablement : AP, t. XIX, p. 579.

27. AP, t. XIX p. 446-578; t. XXI, p. 125-127.

28. Voir également : rapport de Payen concernant les troubles de Martinique, 21 avril 1791, AP, t. XXV, p. 235 ; rapport de Barnave sur la situation des colonies, 23 septembre 1791, t. XXXI, p. 252-259.

29. Moreau avait entrepris de codifier l'ensemble du droit colonial (près de 8000 lois pour SaintDomingue seulement) et avait été engagé à ce titre par le ministre de la Marine, Castries, dans les années 1780. Voir: Dominique TAFFIN (éd.), Moreau de Saint-Méry ou Les ambiguités d'un créole des Lumières : actes du colloque, 10-11 septembre 2004, Fort-de-France, Société des amis des archives et de la recherche sur le patrimoine culturel des Antilles, 2006.

30. AP, t. XXII, p. 138-139.

31. AP, t. XIX, p. 570.

32. AP, t. XXVII, p. 213-231; 236-237.

33. AP. t. XXII, p. 665-666.

34. AP, t. XXII, p. 483.

35. AP, t. XXII, p. 350.

36. Le Patriote français, vol. 3, p. 446-447.

37. Le seul projet législatif notable du Comité, qui concernait la représentation coloniale, fut présenté le 18 août et voté le 22 août 1792. AP, t. XLVIII, p. 358-361.

38. Par exemple, le rapport présenté par Rouyer qui confirme la commission civile envoyée à Saint-Domingue et fait décréter le rappel des commissaires civils aux Iles-du-Vent le 5 novembre 1792. AP, t. LIII, p. 167.

39. AP, t. LIII, p. 685-686, 30 novembre 1792, "acte d'accusation contre Blanchelande »; t. LVII, p. 190, 4 février 1793, rapport relatif «à divers fonctionnaires civils et militaires de SaintDomingue, prévenus d'avoir été les principaux agents du projet de contre-révolution dans les colonies »; t. LX, p. 442, 25 mars 1793, " rapports sur l'affaire des citoyens Brudière et Ligières "; p. 692, 26 mars 1793, « rapports sur l'affaire des citoyens Cambefort et Touzard »; t. LXII, p. 611 « rapport sur la conduite du citoyen Ailhaud»,

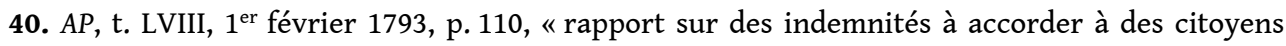
déportés injustement des colonies",; AP, t. LXV, 28 mai 1793, p. 489, "rapport sur les secours à accorder aux déportés de Saint-Domingue ».

41. En particulier, le décret du 19 février 1793 qui ouvrait les ports des Antilles aux Américains, et, surtout, celui du 5 mars - préparé préalablement au sein du Comité colonial - qui mettait en état de défense les colonies et validait la politique menée jusque-là par les commissaires civils à Saint-Domingue.

42. Dans le but de récupérer le local du Comité : A. AULARD, Recueil des actes du Comité de salut public , VI, p. 40.

43. Le rapport du 21 juin 1793 sur les déportés des colonies fut présenté par Poultier au nom des Comités de la guerre, de la marine et des finances réunis, sans participation du Comité colonial : AP, t. LXVII, p. 43. Par le décret d'accusation contre Polverel et Sonthonax du 16 juillet 1793, le Comité de salut public, chargé de faire un rapport pour rétablir l'ordre, avait été autorisé à "prendre au Comité colonial toutes les pièces et renseignements qui pourr[aien]t lui être nécessaires » : AP, t. LXIX, p. 39.

44. Cette commission organisa le grand procès opposant les colons et les commissaires. Elle fut également à l'origine de la série D XXV «Comité des colonies » et du célèbre rapport retraçant la 
révolution de Saint-Domingue. Jean-Philippe GARRAN-DE-COULON, Rapport sur les troubles de SaintDomingue, fait au nom de la commission des colonies, des Comités de Salut public, de Législation et de Marine réunis, Paris, Imprimerie nationale, 1797.

45. Louis-Médéric MOREAU DE SAINT-MÉRY, Sur les dangers de la division du ministère de la Marine et des Colonies, Paris, Impr. nat., 1790.

46. Creuzé-Pascal était un très grand propriétaire de l'île: il se signala notamment par son opposition à la « députation tricolore » de 1794.

47. $\mathrm{AN}, \mathrm{D} \mathrm{XXV}, 89$, "procès-verbaux des séances de la société des colons réunie à l'hôtel Massiac », vol. 4, fo. 55, « $221^{\mathrm{e}}$ séance, 28 avril 1791 », fo. 62 « $222^{\mathrm{e}}$ séance, 3 mai 1791 ».

48. Furent donc adjoints Destutt de Tracy, La Rochefoucauld, Castellanet, Brostaret, PérisseDuluc et Louis Monneron. A part ce dernier, député de Pondichéry mais favorable aux droits des libres de couleur, ces nouveaux membres étaient étrangers aux intérêts coloniaux. AP, t. XXIX, p. 625-628.

49. AP, t. XXX, p. 55-56.

50. Le 11 mai 1791, Barnave avait soutenu aux jacobins un discours contre Brissot et s'y était fait applaudir ; mais le 25 septembre 1791, il en fut exclu suite au débat colonial de la veille, avec les Lameth et Duport sur proposition de Polverel et Régnier : voir L. Deschamps, op. cit., p. 239.

51. G. DEBIEN, op. cit., p. 341.

52. M. Dorigny soutient qu'il faut intégrer Creuzé-Latouche à la mouvance girondine plutôt qu'à la Plaine. Toutefois, sur les questions coloniales, ce député se montre bien plus modéré et discret que ses collègues de Gironde. On sait du reste combien il est difficile de définir l'appartenance girondine. Sur les 12 députés ayant siégé dans le Comité, un membre a été exécuté le 31 octobre avec Brissot, 2 firent partie des 75 protestant contre l'arrestation du 2 juin, 6 furent appelés Girondins. Voir A. soboul (éd.), op. cit., p. 311; Michael John sydenham, The Girondins, Londres, University of London, Athlone press, 1961, p. 218-226.

53. Boyer-Fonfrède afficha son soutien à la commission civile dans le journal de Brissot, deux jours avant la présentation du rapport Camboulas à la Convention le 5 mars 1793. Patriote français, $\mathrm{n}^{\circ} 1299$, p. 257-258.

54. Sur la demande initiale de Mazade lors de la séance du comité du 10 janvier 1793 ; le Comité ne retint en fait qu'une seule accusation, contre le gouverneur d'Esparbès. AN, D*XVI 5, fo. 24-25.

55. AP, t. LVIII, p. 110.

56. F. GAUTHIER, op. cit., p. 141.

57. AP, t. XXIV, p. 595.

58. AP, t. XXII, p. 483; t. XXIV, p. 682; t. XXV, p. 636-638.

59. ANOM, 87 MIOM 95, fo.262-272.

60. Le principal contact en son sein est Martel dont la fréquentation avec Brulley et Page est attestée à partir de juin 1793 mais parait antérieure. AN, D XXV 76, dos. 751, fo. 11.

61. Ils furent tactiquement blanchis le 28 juin 1791, sur proposition du Comité colonial : AP, t. XXVII, p. 583-584.

62. J.-P. GARRAN-DE-COULON, op. cit., 1797, t. I, p. 110.

63. AN, D*XVI 5, fo. 4, séances du 10 et 11 novembre 1791.

64. AN, D XXV 76, dos. 749, «Copies des lettres de Millet, Cougnacq Mion, Chesneaux de la Mesgrière, Lebugnet, La Gourgue, Saint James, Commissaires de l'Assemblée générale de Saint Domingue près la métropole ", non foliotées.

65. AN, D XXV 63, dos. 629, «réponses des commissaires de St. Domingue aux questions du citoyen Boyer Fonfrède membre du comité colonial », 30 janvier 1793.

66. AN, D*XVI 5, fo. 27.

67. AN, D*XVI 2, fo. 31-32, fo 47. Sur le rôle joué par ce décret, voir : J. POPKIN, op. cit., p. 336.

68. ANOM, 87 MIOM 95, fo. 266, 23 mai 1791. 
69. AN, D*XVI 5 , fo. 6.

70. AN, D*XVI 5, fo. 27.

71. AN, D*XVI 2, fo. 31, 26 mars 1793.

72. Augustin-Jean BRULLEY et Pierre-François PAGE, Développement des causes, troubles et désastres des colonies françaises, Paris, 1793, p. 16-17.

73. «quoique nous soyons ici en famille, il ne faut pas se dissimuler que ce qui s'y passe sera connu » : ANOM, 87 MIOM 95, fo. 185, 27 avril 1791.

74. Calculs effectués d'après les registres conservés sous la cote AN, D*XVI 5.

75. Selon Linguet, Annales politiques et littéraires, t. XVIII, $\mathrm{n}^{\circ}$ 158, p. 143.

76. AP, t. XXVII, p. 229-230.

77. G. DEBIEN, op. cit., p. 190.

78. Gonyn, rapport sur la Martinique, (AP, t. LXII, p. 672) ; Lécurel, trois rapports sur des affaires particulières de Tobago, (t. XLI, p. 363 ; XLII, p. 324 ; XLIII, p. 41) ; Levavasseur sur la Guyane et Bourbon (t. XLV, p. 195-196; L, p. 592-609).

79. Rapports sur « les troubles de Saint-Domingue » présentés par Tarbé le 18 novembre 1791 (AP ,t. XXXV, p. 131-136), le 10 décembre 1791 (p. 701-710), le 11 janvier 1792 (t. XXXVII, p. 222-229), le 29 février 1792 (t. XXXIX, p. 198-209); par Journu-Auber le 10 février 1792 (t. XXXVIII, p. 354-358), le 27 mars 1792 (t. XL, p. 533-538), le 15 mai 1792 (t. XLIII p. 424-428).

80. AN, D*XVI 5 , fo. 4.

81. AN, D*XVI 5 , fo. 26.

82. ANOM, 87 MIOM 95, fo. 69,3 février 1791.

83. ANOM, 87 MIOM 95, fo. 238-246, 4 mai 1791.

84. AP, t. XVII, p. 411.

85. AN, D XVI 1.

86. AP, t. XXX, p. 283.

87. ANOM, 87 MIOM 95, séance du 4 février 1791, fo. 72-78.

88. AN, D XXV 76, dos. 749, « Copies des lettres... », 25 décembre 1791

89. AP, t. XXV, p. 472-490.

90. $A P$, t. XXV, p. 645.

91. AN, D XXV 76, dos. 749, « Copies des lettres... », 26 février 1792.

92. Ibid., 20 mars 1792.

93. G. DEBIEN, op. cit., p. 201.

94. AP, t. XXXVII, p. 352.

95. Marcel DORIGNY et Fabrice MATTATIA, «Gaspard Monge dans la Révolution: du ministère girondin au Comité de salut public de l'an II », Bulletin de la Sabix [En ligne], 41, 2007 ; AN, D XXV 76, dos. 750 « Registre des délibérations du Bureau de la Commission de l'assemblée coloniale de la partie française de Saint-Domingue », fo. 32, 34.

96. Jean TARRADE, «L'administration coloniale en France à la fin de l'Ancien Régime : Projets de réformes ", Revue historique, n 229, mars 1963, p. 103-122.

\section{RÉSUMÉS}

Le Comité des colonies sous la Constituante incarna la «corruption » du mouvement patriote. Pourtant composé de "révolutionnaires", il fit obstacle à l'abolition de la traite ainsi qu'à 
l'extension des droits de citoyen actif aux libres de couleur. Son opposition farouche à l'application de la Déclaration des droits de l'homme dans les colonies en fit l'ennemi principal de " philanthropes ", pourtant prudents. L'article vise à aller au-delà de cette image, à juste titre controversée, en étudiant les formes successives du Comité sous les trois premières Assemblées. On peut ainsi observer les transformations d'une institution qui s'appropria des compétences étendues pour tenter d'imposer sa politique coloniale. Loin d'être monolithique toutefois, le Comité fut le lieu d'affrontements multiples, qui opposèrent une grande variété de lobbies coloniaux, et dont le célèbre Club Massiac ne sortit pas toujours gagnant. Soucieux d'échapper aux regards de l'Assemblée mais très perméable à ces groupes de pression, le Comité constitua un lieu de débats, qui devaient néanmoins rester «en famille » - une "famille » se dissimulant derrière le paravent de l'« expertise coloniale ».

The Constituent Assembly's Colonial Committee came to embody the « corruption "of the patriot movement. Though composed of " revolutionaries », it successfully thwarted the abolition of the slave trade and the extension of active citizenship to free people of color. Its fierce opposition to the implementation of the Declaration of Rights in the colonies made it the main enemy of the still-cautious «philanthropists ", This article aims to revise this controversial image by analyzing the successive forms taken by the committee under the three assemblies. The study sheds new light on a transforming institution that managed to amass considerable power and endeavored, with greater and lesser success, to impose its own colonial policy. The committee, however, was far from monolithic: it was the site of numerous struggles among colonial lobbies, including the famous Club Massiac. Desirous of escaping the surveillance of the Assembly but very susceptible to influence from interest groups, the Committee became a site of debates. However, these debates were supposed to stay within the "family» circle-a family that concealed itself behind the screen of « colonial expertise ».

\section{INDEX}

Mots-clés : Comité des colonies, Révolution française, colonies, lobby colonial

Keywords : Colonial Committee, French Revolution, Colonies, colonial lobbies

\section{AUTEUR}

MANUEL COVO

Doctorant (CENA-MASCIPO, EHESS)

ATER (Centre Georges Chevrier, Université de Bourgogne) 